\title{
Theoretical Determination of Interaction and Cohesive Energies of Weakly Bound Cycloparaphenylene Molecules
}

\author{
M. Reche-Tamayo ${ }^{1}$, M. Moral ${ }^{1,2}$, \\ A. J. Pérez-Jiménez ${ }^{1}$, and J. C. Sancho-García ${ }^{1 *}$ \\ ${ }^{1}$ Departamento de Química Física, \\ Universidad de Alicante, E-03080 Alicante, Spain \\ ${ }^{2}$ Instituto para la Investigación en Energías Renovables, \\ Universidad de Castilla-La Mancha, E-02071 Albacete, Spain
}

September 5, 2016

*E-mail: jc.sancho@ua.es; Phone number: +34 / 965903536 


\begin{abstract}
We investigate here, by applying dispersion-corrected theoretical methods, the energy stability of dimers formed by $[n]$ cycloparaphenylene molecules $(n=5,6,7,8,10$, and 12 being the number of benzene rings strained to form the nanoring) when they self-assemble in crystalline samples. Their cyclic topology confers to these samples a rich variety of dimer orientations, i.e. tubular or herringbone-like, according to the nanoring size, with the final form of their crystal packing depending subtly on the energy difference and the number of symmetry-related repetitions between these two microstructures. We finally calculate the cohesive energies for the illustrative cases $n=6$ and $n=12$, through the interaction energies of the unique and symmetry-related supramolecular motifs found, to finally understand the driving forces between the emergence of nanochannel-like structures.
\end{abstract}




\section{Introduction}

Nanoforms based on $\mathrm{sp}^{2}$ carbon backbones are attracting now a tremendous interest worldwide. ${ }^{1}$ Actually, fullerenes, nanocones, nanographenes, nanofoams, or nanotubes, to name just a few of them, constitute a large family of compounds with many envisioned possibilities for further functionalization. ${ }^{2}$ For the use of these materials in the wide range of large-scale and real-world expected applications, these forms need to be produced in industrial quantities, at a controlled scale and free of impurities, with some further automation and scaling-up, and with the desired selectivity of the final products arising from their synthesis. For instance, carbon nanostructures are not soluble in many media, and non-conventional alternative strategies are always under development. ${ }^{3,4}$ However, this is not always the case for SingleWalled Carbon NanoTubes (SWCNTs), for which a concise bottom-up synthesis toward customized structures (i.e. fine-tuning their shape, size, and topology) may still need further achievements for solution-based processes. ${ }^{5,6}$

Within this context, one of the most successful and timely approaches has been the use of molecular templates for the growth of size-defined SWCNTs, using chemical precursors for that such as CycloParaPhenylene (CPP) molecules,${ }^{7}$ synthesized by the first time in $2008,{ }^{8}$ or other envisioned molecules acting as CNT segments such as beltenes, ${ }^{9}$ collarenes, ${ }^{10}$ cyclacenes, ${ }^{11}$ calixarenes, ${ }^{12}$ or cyclopyrenes, ${ }^{13}$ to name just a few of them. ${ }^{14,15}$ All of these systems share that are formed upon progressively bending some aromatic units to form a cyclic nanoring. The particular case of a phenylene moiety gives rise to the cyclic topology presented in Figure 1, where the number $n$ of units control their final size. ${ }^{16-19}$ 
The hypothetical self-assembly of the latter templates, based on $\mathrm{C}_{2} \mathrm{H}$ additions and subsequent hydrogen abstraction, has been theoretically described in some detail, ${ }^{20}$ which might pave the way toward a mechanism for the growth of SWCNT with fine-tuned diameters. ${ }^{21}$ Therefore, the longitudinal growth of these CPPs precursors is a very promising alternative, ${ }^{22}$ although is still not free from experimental difficulties due to the expected formation of some cationic species and then reactive intermediates. ${ }^{23}$ However, recent progress are also made to disclose new synthetic routes, with short reaction steps and high yields. ${ }^{24}$

Complementarily to the fine-tuning of challenging synthetic routes, one would benefit from understanding and rationalizing the energy stability of weakly bound CPPs molecules, because they are known to spontaneously self-assemble in the solid-state. In fact, the molecular arrangements found in their crystalline state show very directional and close intermolecular contacts, with varying molecule-to-molecule orientations ${ }^{25}$ depending on their size $n$. Therefore, finding out which interactions are favored with respect to others may help to ascertain the growth or elongation paths that can be further exploited.

To this end, we will investigate in the following the energy of all existing dimers (i.e. two interacting molecules extracted from crystalline samples of $[n] \mathrm{CPP})$ by means of accurate computational methods taking into account the non-covalent forces operating between the interacting entities. We devote the following section to underline the main features of the theoretical methods used, to tackle next the calculation of supramolecular (i.e. dimer) interaction energies, which will we subsequently used to estimate cohesive 
energies and rationalize further their crystalline structures.

\section{Computational details}

The structure of all the unique dimers considered is extracted from their respective crystalline forms, and used rigidly herein. We processed the corresponding files with the Mercury program. ${ }^{26}$ Solvent molecules were conveniently removed in [7]CPP and [12]CPP cases to exclusively investigate the non-covalent interactions between chemically relevant pairs of $[\mathrm{n}] \mathrm{CPP}$ molecules. These solvent molecules were always found occluded within the cavity of the compounds, and are thus not expected to greatly affect the conclusions reached here for the studied intermolecular interactions between neighbouring dimers in the crystal. The intermolecular interaction energy of every dimer, $\Delta E_{\text {int }}$, which depends upon the mutual geometrical arrangement of the isolated subunits or monomers, is calculated by subtracting the energy of both monomers at the dimer geometry $\left(Q^{\text {dimer }}\right)$ to that of the interacting dimer: ${ }^{27}$

$$
\Delta E_{\text {int }}=E_{\text {dimer }}\left(Q^{\text {dimer }}\right)-2 E_{\text {monomer }}\left(Q^{\text {monomer }} / / Q^{\text {dimer }}\right),
$$

with a negative value thus implying a bound dimer stabilized mostly by weak (i.e., non-covalent) interactions. We efficiently take into account all existing, intra- and intermolecular, non-covalent interactions by resorting to the B3LYP-D3(BJ) dispersion-corrected functional, in which a pairwise correction -D3(BJ) ${ }^{28,29}$ is added to the electronic B3LYP energy ${ }^{30,31}$ of both dimer and monomer subunits. The form of the correction is given by:

$$
E_{\mathrm{D} 3}(A, B)=-\sum_{n=6,8} s_{n} \sum_{B>A}^{\text {atomic-pairs }} \frac{C_{n}^{A B}}{R_{A B}^{n}} f_{n}\left(R_{A B}\right)
$$


where $A$ and $B$ represent all interacting atoms within the dimer, $R_{A B}$ are the corresponding distances, $C_{n}^{A B}$ are the $n$ th-order interatomic dispersion coefficients, $s_{n}$ are functional-dependent parameters, and $f_{n}\left(R_{A B}\right)$ is a damping function able to switch the above energy contribution from short- to longrange interatomic distances. This way of adding the non-covalent energy, $E_{\mathrm{D} 3}$ at a fixed structure, to the electronic energy self-consistently computed with the B3LYP method, has itself revealed as a very efficient and accurate method. ${ }^{32}$ We will use in the following the large cc-pVTZ basis set, to avoid as much as possible basis set incompleteness issues such as the Basis Set $\mathrm{Su}$ perposition Error, and will increase all the default numerical thresholds (e.g. Int $=$ Ultrafine). The calculations were done with the Gaussian 09 package. ${ }^{33}$

\section{Results and discussion}

\subsection{Supramolecular interaction energies}

Table 1 gathers the relevant unit cell parameters of the $[n] \mathrm{CPP}$ compounds considered here, as well as the averaged diameter of the corresponding nanorings. The calculated interaction energies for all the $[n] \mathrm{CPP}(n=$ $5,6,7,8,10$, and 12) dimers studied, extracted from the specific crystallographic files (vide infra), are shown in Figure 2 for the sake of a rapid visual inspection. Each subfigure $2 \mathrm{a}-2 \mathrm{f}$ is devoted to each $[n] \mathrm{CPP}$ compound, and displays the energy profile (from the highest to the lowest stability) for all the existing dimers. These values are numerically provided in Table 2, following the same order than in Figure 2. The evolution and magnitude of these interaction energies allow us to briefly underline here some of the main findings:

- The herringbone ("T"- or "V"-shaped) driven pattern seems to be en- 
ergetically unfavored with respect to the tubular-like form, with the exception of the [5]CPP case. In all the other cases studied, $n=6$, $7,8,10$, and 12 , a slightly slipped tubular-like arrangement is clearly preferred for most of the dimers, thus showing the lowest interaction energies across the set of microstructures analyzed. This issue may have a tremendous influence on the crystal growth and engineering in the case of $[6] \mathrm{CPP}$.

- For each one of the $[n] \mathrm{CPP}$ systems tackled, even for the smallest and most strained such as the set of $[5-8] \mathrm{CPP}$ nanorings, there exists at least one microstructure with an interaction energy below $-11 \mathrm{kcal} / \mathrm{mol}$, which can also reach values as large as $-17 \mathrm{kcal} / \mathrm{mol}$ $([10] \mathrm{CPP})$ and $-18 \mathrm{kcal} / \mathrm{mol}([12] \mathrm{CPP})$, and thus with a remarkable stability.

- For the [5] CPP ([6]CPP) case, the lowest-energy dimer exceeds by $53 \%$ (55\%) the following energy value. This excess are much less pronounced for the rest of the compounds, which would help to explain the particular solid-state form found for the [6]CPP samples.

\subsection{1 [5]CPP}

The synthesis of the smallest $\left(\mathrm{C}_{30} \mathrm{H}_{20}\right)$ member of the family, with a diameter of $6.8 \AA$ and then highly strained, was recently accomplished ${ }^{34}$ and its (refined) crystalline structure concomitantly obtained. ${ }^{35}$ One of the most striking features of this solid-state structure, as it was emphasized by the own authors of Ref. 352014Evans et al.Evans, Darzi, and Jasti, was the adoption of a shallow (boat-like) conformation by the individual molecules, which is clearly observed in the microstructures shown in Figure 2a for this molecule. The herringbone-like dimer is the most stable one, with an interaction en- 
ergy of $-12.01 \mathrm{kcal} / \mathrm{mol}$, approximately twice the value of the following microstructures (with interaction energies of -6.32 and $-5.95 \mathrm{kcal} / \mathrm{mol}$, see Figure 2) and far from the $-3.50 \mathrm{kcal} / \mathrm{mol}$ of the weakest bound dimer.

\section{$3.1 .2[6] \mathrm{CPP}$}

Interestingly, and contrarily to what happened for $[5] \mathrm{CPP}$, the $[6] \mathrm{CPP}$ compound $\left(\mathrm{C}_{36} \mathrm{H}_{24}\right)$ self-assembles in a tubular packing, which might pave the way toward further crystal engineering. The synthesis and following recrystallization of the [6]CPP samples led to the unit cell parameters included in Table 1 for an hexagonal $R-3$ space group. ${ }^{36}$ The strain enthalphy, that is the energy needed to close the para-phenylene units into the corresponding nanoring, calculated at the B3LYP-D3(BJ)/6-31+G* level, decreases from

$108.6([5] \mathrm{CPP})$ to 89.8 ([6] CPP) $\mathrm{kcal} / \mathrm{mol},{ }^{37}$ together with an increase in the diameter from 6.8 to $8.4 \AA$. The density of the solid-state samples decreases from $1.243([5] \mathrm{CPP})$ to $1.126([6] \mathrm{CPP}) \mathrm{Mg} \cdot \mathrm{m}^{-3}$ respectively. These (apparently small) changes dramatically alter the packing pattern, with a tubular-like dimer being now the most stable one having an interaction energy of $-14.21 \mathrm{kcal} / \mathrm{mol}$, see Figure $2 \mathrm{~b}$, followed by parallel arrangement of a (almost degenerate) pair of molecules (with interaction energies of -7.90 and $-7.86 \mathrm{kcal} / \mathrm{mol}$ ) and the corresponding out-of-plane diagonal dimer (with the lowest interaction energy of $-4.60 \mathrm{kcal} / \mathrm{mol}$ ). Note also how the number of unique dimers is reduced with respect to the previous case, without any microstructure now adopting a herringbone shape. 


\subsection{3 [7]CPP}

The selective synthesis of [7]CPP was also recently accomplished ${ }^{38}$ and extended to a large set of $[n] \mathrm{CPP}$ compounds. ${ }^{39}$ The solid-state samples, although the compound could only be crystallized with stoichiometry $\mathrm{C}_{48} \mathrm{H}_{40},{ }^{40}$ intriguingly recover the herringbone orientation. The interaction energies amounts now to $-11.31,-9.35,-8.67$, and $-5.87 \mathrm{kcal} / \mathrm{mol}$, respectively, for the set of dimers included in Figure 2c. Once again, the dimer with a tubular-like orientation recovers the highest interaction energy among the set of microstructures, although energetically separated from the next of the dimers (herringbone) by only around $2 \mathrm{kcal} / \mathrm{mol}$.

\subsection{4 [8]CPP}

The gram-scale synthesis of $[8] \mathrm{CPP}, \mathrm{C}_{48} \mathrm{H}_{32}$, and its subsequent recrystallization from dichloromethane-hexane solutions, led again to herringbone structures. ${ }^{41}$ Figure $2 \mathrm{~d}$ gathers all the dimers analyzed, with interaction energies (in decrease order) of $-14.04,-10.72,-10.40$, and $-6.42 \mathrm{kcal} / \mathrm{mol}$, respectively. Additionally to the herringbone pattern, we also see highly stable quasi-1D tubular-like microstructures. Interestingly, the herringbone dimer is energetically separated by only around $3 \mathrm{kcal} / \mathrm{mol}$ from the tubularlike example.

\subsection{5 [10]CPP}

The crystal structure of [10] CPP $\left(\mathrm{C}_{60} \mathrm{H}_{40}\right)$ did not differ too much from that of $[8] \mathrm{CPP},{ }^{41}$ providing that the unit cell parameters are $a=15.9075 \AA$, $b=8.1405 \AA, c=20.7418 \AA, \alpha=\gamma=90^{\circ}, \beta=108.655$, for an mon- 
oclinic space group, with the dimers shown in Figure 2e. The interaction energies are now $-17.32,-14.38,-9.39$, and $-6.98 \mathrm{kcal} / \mathrm{mol}$, showing again an energy preference for the tubular-like dimer, closely followed by the dimer representative of the herringbone pattern distant only around $3 \mathrm{kcal} / \mathrm{mol}$ in energy, and with the rest of the dimer separated by more than $5 \mathrm{kcal} / \mathrm{mol}$.

\subsection{6 [12]CPP}

In 2011, the first crystal structure of [12] CPP was presented, ${ }^{42}$ although cocrystallized in the form [12]CPP.cyclohexane $\left(\mathrm{C}_{84} \mathrm{H}_{72}\right)$. In addition to the herringbone dimer, with an interaction energy of -16.14 , two [12]CPP molecules also align in a tubular-like form, displaying again the lowest interaction energy $(-18.47 \mathrm{kcal} / \mathrm{mol})$ among all the microstructures, although separated energetically by around $2 \mathrm{kcal} / \mathrm{mol}$ from the former one. The next supramolecular orientations appear far in energy, at $-7.98 \mathrm{kcal} / \mathrm{mol}$ for the parallel-like case, followed by two nearly degenerate dimers with interaction energies of $-7.49 \mathrm{kcal} / \mathrm{mol}$.

\subsection{Cohesive energies}

The cohesive or lattice energy, $U$, is defined usually as the amount of energy required to separate a mole of the solid into a gas of its constituent molecules. We will estimate it here through the sum of energies of all interacting yet unique molecular pairs,

$$
U=-\frac{1}{2} \sum_{i}^{\text {unique dimers }} m_{i} \Delta E_{\mathrm{int}}^{(i)}
$$

being $m_{i}$ the number of unique pairs in which the supramolecular sample

can be decomposed, and $\Delta E_{\text {int }}^{(i)}$ the interaction energy for each dimer $i$ as 
calculated by Eq. (1). The result must be divided by two to avoid the double-counting. ${ }^{43}$ Despite its simplicity, the use of this expression keeps some interesting features since it would allow: (i) to easily compare the results here with previous estimates for other PAH (Polyciclic Aromatic Hydrocarbons) ${ }^{44-46}$ and (ii) to disentangle the relative contribution of each dimer to the total value. ${ }^{47}$ This energy, made positive, approximates the sublimation enthalphy at room temperature $(298.15 \mathrm{~K})$ by $U=\Delta_{s} H(T)+2 R T$, with $2 R T$ being the (classical) thermal contribution. Note also that we will restrict in the following to the interactions within the first shell, and assume a pairwise character of intermolecular forces.

We will focus on the [6]CPP and [12]CPP cases, since they are believed to represent paradigmatic cases. The crystal structures were obtained, respectively, from Refs. 362012Xia and JastiXia, and Jasti and 422011Segawa et al.Segawa, Miyamoto, Omachi, Matsuura, Šenel, Sasamori, Tokitoh, and Itami. Whereas the [6]CPP array of molecules forms completely different packing motifs with respect to the rest of systems, as it was emphasized previously, the latter is also known to display a tubular-like structure along one axis, with some envisioned use for microencapsulation of size-complementary fullerene molecules ${ }^{48-50}$ or as a new porous-based nanomaterial with unique adsorption behavior. ${ }^{51}$ Furthermore, they may promisingly behave as efficient ambipolar semiconductors in solid-state devices, ${ }^{52}$ and might serve as a template for further crystal engineering. Thus, supercells are constructed from the experimental units cells, with the corresponding crystal structures displayed in Figures 3 and 4, respectively, from which one can infer the value of $m_{i}$ after looking at the number of dimer interactions from a molecule chosen as reference. For [6]CPP, and going from left to right into the rows of 
Table 2 gathering the interaction energies, $m_{i}$ equals to 2,2 , 4, and 6 , respectively, for the tubular-like, laterally interacting and diagonal dimers. This is also the order (from left to right) followed in Figure 2b. Feeding now Eq. (3) with these values, it leads to a cohesive energy $U=51.63 \mathrm{kcal} / \mathrm{mol}$, considerably high as compared with other polycyclic aromatic hydrocarbons. ${ }^{53}$ If we switch now to the [12] CPP supramolecular structure, $m_{i}=2$ in all cases, it leads to a cohesive energy $U=57.55 \mathrm{kcal} / \mathrm{mol}$, and thus slightly higher than that obtained for $[6] \mathrm{CPP}$.

\subsection{Rationalization of the results}

There exists a set of models to predict sublimation (or lattice) energies of compounds from their molecular structure (or connectivity) and/or their molecular properties. For instance, some specific equations derived for aromatic hydrocarbons using only atom types and their bonded environment, ${ }^{54}$ which is our case reduce to the simple form $\Delta_{\text {sub }} H=4.162+6.185 C_{\text {aromatic }}$ $(\mathrm{kJ} / \mathrm{mol})$ with $C_{\text {aromatic }}$ the number of $\mathrm{C}$ atoms involved in an aromatic system, allow us to easily predict $U$ values for a compound with molecular formula $\mathrm{C}_{36} \mathrm{H}_{24}$ (i.e. [6] CPP) between $54-56 \mathrm{kcal} / \mathrm{mol}$, with minor variations due to the effect of using one or another type of the proposed multilinear regressions in Ref. 542003Ouvrard and MitchellOuvrard, and Mitchell, and between $106-109 \mathrm{kcal} / \mathrm{mol}$ for the corresponding $\mathrm{C}_{72} \mathrm{H}_{48}$ (i.e. [12]CPP). Note that: (i) the models used here, despite its simplicity, have sufficiently been validated before and estimate values usually within deviations of $10 \%$ or less with respect to experimental data of organic compounds; (ii) the value found here for $[6] \mathrm{CPP}$ (i.e. $U=51.63 \mathrm{kcal} / \mathrm{mol}$ ), after the calculations performed at the B3LYP-D3(BJ)/cc-pVTZ level, fits well into the prediction of 
the model equation selected for aromatic hydrocarbons, that is $U=55.49$ $\mathrm{kcal} / \mathrm{mol}$, with a relative error roughly of $7 \%$; (iii) however, this is not the case for [12]CPP, where the calculated value of $U$ at the B3LYP-D3(BJ)/ccpVTZ level, $57.55 \mathrm{kcal} / \mathrm{mol}$, significantly deviated from the prediction of the trained equations used (i.e. $U=108.62 \mathrm{kcal} / \mathrm{mol}$ ); and (iv) the values predicted for the linear analogues by employing these models for the molecules ( $p$-terphenyl and $p$-quaterphenyl) for which experimental sublimation enthalpies are known, agree fairly well (within $5-10 \%$ ) with respect to their experimental counterparts.

Actually, and contrarily to their cyclic analogues, the para-phenylene systems always crystallize in a herringbone fashion, independently of their system size. The dimer typical of a herringbone crystal packing appears actually for the smallest $([5] \mathrm{CPP})$ case studied here, being uninterrumpedly one of the low-energy dimers up to the $[12] \mathrm{CPP}$ compound, with the notable exception of [6]CPP. The energy separation of the herringbone-like dimer, for the set of $[n] \mathrm{CPP}$ samples studied, remains only $2-3 \mathrm{kcal} / \mathrm{mol}$ higher with respect to the lowest-energy one, with two remarkable exceptions: (i) in the [5]CPP case, the energy order is reversed, with the herringbone-like dimer much more stable in energy; and (ii) the herringbone dimer does not even appear for $[6] \mathrm{CPP}$, thus reducing the total number of dimers around the crystal axis. It thus seems that the type and strength of packing for the $[n] \mathrm{CPP}$ compounds is not finally determined by the nature of the moleculeto-molecule interactions, but by the optimal space filling across the samples.

If we weight now the relative contribution of each dimer (i.e. crystallographic direction of growing) to the cohesive energy, by using a simple 
expression such as:

$$
W=\frac{m_{i} \Delta E_{\mathrm{int}}^{(i)}}{\sum_{i} m_{i} \Delta E_{\mathrm{int}}^{(i)}},
$$

we can estimate, neglecting kinetic and disorder effects, the percentage given by every dimer to the cohesive energy. In the case of $[6] \mathrm{CPP}$, the tubular dimer contributes to $27.5 \%$, the parallel-like with $45.7 \%$, and the diagonal with $26.7 \%$, indicating a slightly biased preference for a layered growth concomitantly with an epitaxial mechanism. On the other hand, the corresponding values for [12]CPP are $32.1 \%$ for the tubular-like and $28.0 \%$ for the herringbone, largely separated from the rest of dimer interactions. These values allow us to ascertain a concerted 2D-like growth, possibly with a strong interplay between the nanochannel-like direction and the herringbone interactions at both sides of it.

With this information in mind, we therefore look at the experimental solid-state density of samples of both cyclic (i.e. $[n]$ CPP) and linear (i.e. $[n] \mathrm{PP})$ compounds. This density smoothly decreases as a function of the system size for $[n] \mathrm{CPP}$ compounds (see Table 1 ). Neglecting those samples with cocrystallized solvent molecules (normally cyclohexane or dichloromethane) the density goes from a value of $1.243([5] \mathrm{CPP})$ to $0.993([10] \mathrm{CPP}) \mathrm{Mg} \cdot \mathrm{m}^{-3}$. On the other hand, the density of the $[5-7] \mathrm{PP}$ compounds only slightly fluctuates around a value of $1.30 \pm 0.01 \mathrm{Mg} \cdot \mathrm{m}^{-3}$, being already higher than for any of the $[5-12] \mathrm{CPP}$ crystalline samples, clearly indicating the amount of void space left inside the channels in the crystal packing of $[n]$ CPPs compared to the linear forms.

Admittedly, all these findings reveal how the cyclic topology of the $[n] \mathrm{CPP}$ compounds becomes the key factor to become outliers of the trend found be- 
tween sublimation enthalpies and the molar mass, and to keep interesting structure-property relationships despite this fact. Due to the strong influence of intermolecular interactions in the sublimation process, and also due to its pairwise nature in dispersion-bound complexes, it is clear that its value should increase with molecular size. But interactions between the molecules and the air plays also a dominant role, and properties as the surface polarity are expected to significantly affect the values. ${ }^{55}$ Contrarily to their linear analogues, the cyclic nature of $[n] \mathrm{CPP}$ precludes the optimal space filling, and then highest possible values according to their chemical formula $\mathrm{C}_{6 n} \mathrm{H}_{4 n}$.

\section{Conclusions}

The cyclic topology of the $n$-ring oligomers of CPP critically determines their crystalline packing. When this packing is analyzed in much more detail through all the existing structural dimers, we found some regularity for the corresponding microstructures (that is the orientation between the pair of interacting molecules zoomed in) despite the different unit cell parameters and the space groups to which the compounds belong to.

We have systematically calculated the non-covalent association energy for all existing dimers, using a robust dispersion-corrected DFT method with large basis sets. The herringbone pattern is present in for all cases (except for [6] CPP) which is typical of the packing of other polyciclic aromatic hydrocarbons, particularly for the linear analogues of the systems studied here. The tubular pattern, two superimposed (although slightly slipped) molecules with the shortest $\mathrm{C}-\mathrm{C}$ separation roughly around $4 \AA$, is also found in all cases, except for [5]CPP. This dimer is found here to be the lowest in energy 
independently of the oligomer size.

We have also calculated the cohesive energy for [6]CPP and [12]CPP, assuming a pairwise additivity of the calculated energy of association for the weakly bound dimers. The value for the latter system is $12 \%$ higher with respect to the former, despite having twice its molar mass $\left(\mathrm{C}_{72} \mathrm{H}_{48}\right.$ vs. $\mathrm{C}_{36} \mathrm{H}_{24}$ ). However, the density of the crystalline samples of $[n] \mathrm{CPP}$ significantly decreases with the oligomer size, as a consequence of the increase in the diameter. Thus, the interplay between the non-covalent forces operating between molecules within the unit cell, and the amount of void space left inside the nanochannels, seems to finally dominate the whole supramolecular packing and the associated cohesive energy.

\section{Acknowledgements}

This work is supported by the "Ministerio de Economía y Competitividad" of Spain and the "European Regional Development Fund" through project CTQ2014-55073-P. MMM thanks the E2TP-CYTEMA-SANTANDER program for financial support and the High Performance Computing Service of University Castilla-La Mancha for technical support.

\section{References}

[1] Segawa, Y.; Yagi, A.; Matsui, K.; Itami, K. Design and Synthesis of Carbon Nanotube Segments. Angewandte Chemie International Edition 2016, 55, 5136-5158. 
[2] Delgado, J. L.; Herranz, M. Á.; Martin, N. The nano-forms of carbon. Journal of Materials Chemistry 2008, 18, 1417-1426.

[3] Vázquez, E.; Giacalone, F.; Prato, M. Non-conventional methods and media for the activation and manipulation of carbon nanoforms. Chemical Society Reviews 2014, 43, 58-69.

[4] Mateos-Gil, J.; Rodríguez-Pérez, L.; Oliva, M. M.; Katsukis, G.; Romero-Nieto, C.; Herranz, M. Á.; Guldi, D. M.; Martín, N. Electroactive carbon nanoforms: a comparative study via sequential arylation and click chemistry reactions. Nanoscale 2015, 7, 1193-1200.

[5] Golling, F. E.; Quernheim, M.; Wagner, M.; Nishiuchi, T.; Müllen, K. Concise Synthesis of 3D $\pi$-Extended Polyphenylene Cylinders. Angewandte Chemie International Edition 2014, 53, 1525-1528.

[6] Golling, F. E.; Osella, S.; Quernheim, M.; Wagner, M.; Beljonne, D.; Müllen, K. $\pi$-extended [12]cycloparaphenylenes: from a hexaphenylbenzene cyclohexamer to its unexpected $\mathrm{C}_{2}$-symmetric congener. Chemical Science 2015, 6, 7072-7078.

[7] Omachi, H.; Nakayama, T.; Takahashi, E.; Segawa, Y.; Itami, K. Initiation of carbon nanotube growth by well-defined carbon nanorings. Nature Chemistry 2013, 5, 572-576.

[8] Jasti, R.; Bhattacharjee, J.; Neaton, J. B.; Bertozzi, C. R. Synthesis, characterization, and theory of [9]-,[12]-, and [18] cycloparaphenylene: carbon nanohoop structures. Journal of the American Chemical Society 2008, 130, 17646-17647.

[9] Choi, H. S.; Kim, D.; Tarakeshwar, P.; Suh, S. B.; Kim, K. S. A New Type of Ionophore Family Utilizing the Cation-Olefinic $\pi$ Interaction: 
Theoretical Study of [n]Beltenes. The Journal of Organic Chemistry 2002, 67, 1848-1851.

[10] Choi, H. S.; Suh, S. B.; Cho, S. J.; Kim, K. S. Ionophores and receptors using cation- $\pi$ interactions: Collarenes. Proceedings of the National Academy of Sciences 1998, 95, 12094-12099.

[11] Chen, Z.; Jiang, D.-e.; Lu, X.; Bettinger, H. F.; Dai, S.; Schleyer, P. v. R.; Houk, K. N. Open-shell singlet character of cyclacenes and short zigzag nanotubes. Organic Letters 2007, 9, 5449-5452.

[12] Kim, K. S.; Suh, S. B.; Kim, J. C.; Hong, B. H.; Lee, E. C.; Yun, S.; Tarakeshwar, P.; Lee, J. Y.; Kim, Y.; Ihm, H. e. a. Assembling phenomena of calix [4] hydroquinone nanotube bundles by one-dimensional short hydrogen bonding and displaced $\pi-\pi$ stacking. Journal of the American Chemical Society 2002, 124, 14268-14279.

[13] Iwamoto, T.; Kayahara, E.; Yasuda, N.; Suzuki, T.; Yamago, S. Synthesis, Characterization, and Properties of [4]Cyclo-2,7-pyrenylene: Effects of Cyclic Structure on the Electronic Properties of Pyrene Oligomers. Angewandte Chemie International Edition 2014, 53, 6430-6434.

[14] Yagi, A.; Venkataramana, G.; Segawa, Y.; Itami, K. Synthesis and properties of cycloparaphenylene-2,7-pyrenylene: a pyrene-containing carbon nanoring. Chemical Communications 2014, 50, 957-959.

[15] Segawa, Y.; Yagi, A.; Ito, H.; Itami, K. A Theoretical Study on the Strain Energy of Carbon Nanobelts. Organic Letters 2016, 18, 14301433.

[16] Omachi, H.; Segawa, Y.; Itami, K. Synthesis of cycloparaphenylenes 
and related carbon nanorings: a step toward the controlled synthesis of carbon nanotubes. Accounts of Chemical Research 2012, 45, 1378-1389.

[17] Yamago, S.; Kayahara, E.; Iwamoto, T. Organoplatinum-Mediated Synthesis of Cyclic $\pi$-Conjugated Molecules: Towards a New Era of ThreeDimensional Aromatic Compounds. The Chemical Record 2014, 14, 84100.

[18] Golder, M. R.; Jasti, R. Syntheses of the smallest carbon nanohoops and the emergence of unique physical phenomena. Accounts of Chemical Research 2015, 48, 557-566.

[19] Lewis, S. E. Cycloparaphenylenes and related nanohoops. Chemical Society Reviews 2015, 44, 2221-2304.

[20] Li, H.-B.; Page, A. J.; Irle, S.; Morokuma, K. Theoretical Insights into Chirality-Controlled SWCNT Growth from a Cycloparaphenylene Template. ChemPhysChem 2012, 13, 1479-1485.

[21] Jasti, R.; Bertozzi, C. R. Progress and challenges for the bottom-up synthesis of carbon nanotubes with discrete chirality. Chemical Physics Letters 2010, 494, 1-7.

[22] Quernheim, M.; Golling, F. E.; Zhang, W.; Wagner, M.; Räder, H.-J.; Nishiuchi, T.; Müllen, K. The Precise Synthesis of Phenylene-Extended Cyclic Hexa-peri-hexabenzocoronenes from Polyarylated [n] Cycloparaphenylenes by the Scholl Reaction. Angewandte Chemie International Edition 2015, 54, 10341-10346.

[23] Sisto, T. J.; Zakharov, L. N.; White, B. M.; Jasti, R. Towards piextended cycloparaphenylenes as seeds for CNT growth: investigat- 
ing strain relieving ring-openings and rearrangements. Chemical Science 2016, 7, 3681-3688.

[24] Patel, V. K.; Kayahara, E.; Yamago, S. Practical Synthesis of [n]Cycloparaphenylenes $(\mathrm{n}=5,7-12)$ by $\mathrm{H}_{2} \mathrm{SnCl}_{4}$-Mediated Aromatization of 1,4-Dihydroxycyclo-2,5-diene Precursors. Chemistry-A European Journal 2015, 21, 5742-5749.

[25] Darzi, E. R.; Jasti, R. The dynamic, size-dependent properties of [5][12] cycloparaphenylenes. Chemical Society Reviews 2015, 44, 64016410 .

[26] Macrae, C. F.; Bruno, I. J.; Chisholm, J. A.; Edgington, P. R.; McCabe, P.; Pidcock, E.; Rodriguez-Monge, L.; Taylor, R.; Streek, J. v.; Wood, P. A. Mercury CSD 2.0-new features for the visualization and investigation of crystal structures. Journal of Applied Crystallography 2008, 41, 466-470.

[27] Scaranto, J.; Mallia, G.; Harrison, N. An efficient method for computing the binding energy of an adsorbed molecule within a periodic approach. The application to vinyl fluoride at rutile $\mathrm{TiO}_{2}(110)$ surface. Computational Materials Science 2011, 50, 2080-2086.

[28] Grimme, S.; Antony, J.; Ehrlich, S.; Krieg, H. A Consistent and Accurate Ab Initio Parametrization of Density Functional Dispersion Correction (DFT-D) for the 94 Elements H-Pu. The Journal of Chemical Physics 2010, 132, 154104.

[29] Grimme, S.; Ehrlich, S.; Goerigk, L. Effect of the damping function in dispersion corrected density functional theory. Journal of Computational Chemistry 2011, 32, 1456-1465. 
[30] Becke, A. D. Density-Functional Thermochemistry. III. The Role of Exact Exchange. The Journal of Chemical Physics 1993, 98, 5648-5652.

[31] Barone, V.; Adamo, C. Theoretical study of direct and water-assisted isomerization of formaldehyde radical cation. A comparison between density functional and post-Hartree-Fock approaches. Chemical Physics Letters 1994, 224, 432-438.

[32] Grimme, S.; Hansen, A.; Brandenburg, J. G.; Bannwarth, C. Dispersioncorrected mean-field electronic structure methods. Chemical Reviews 2016, 116, 5105-5154.

[33] Frisch, M. J. et al. Gaussian 09 Revision E.01. Gaussian Inc. Wallingford CT 2009.

[34] Kayahara, E.; Patel, V. K.; Yamago, S. Synthesis and Characterization of [5]Cycloparaphenylene. Journal of the American Chemical Society 2014, 136, 2284-2287.

[35] Evans, P. J.; Darzi, E. R.; Jasti, R. Efficient room-temperature synthesis of a highly strained carbon nanohoop fragment of buckminsterfullerene. Nature Chemistry 2014, 6, 404-408.

[36] Xia, J.; Jasti, R. Synthesis, Characterization, and Crystal Structure of [6]Cycloparaphenylene. Angewandte Chemie International Edition 2012, 51, 2474-2476.

[37] Climent-Medina, J.-V.; Pérez-Jiménez, Á.-J.; Moral, M.; San-Fabián, E.; Sancho-García, J.-C. Intra-and Intermolecular Dispersion Interactions in [n]Cycloparaphenylenes: Do They Influence Their Structural and Electronic Properties? ChemPhysChem 2015, 16, 1520-1528. 
[38] Sisto, T. J.; Jasti, R. Overcoming molecular strain: synthesis of [7]cycloparaphenylene. Synlett 2012, 2012, 483-489.

[39] Darzi, E. R.; Sisto, T. J.; Jasti, R. Selective syntheses of [7]-[12]cycloparaphenylenes using orthogonal Suzuki-Miyaura crosscoupling reactions. The Journal of Organic Chemistry 2012, 77, 66246628.

[40] Sibbel, F.; Matsui, K.; Segawa, Y.; Studer, A.; Itami, K. Selective synthesis of [7] and [8]cycloparaphenylenes. Chemical Communications 2014, 50, 954-956.

[41] Xia, J.; Bacon, J. W.; Jasti, R. Gram-Scale Synthesis and Crystal Structures of [8] and [10]CPP, and the Solid-State Structure of $\mathrm{C}_{60^{-}}[10] \mathrm{CPP}$. Chemical Science 2012, 3, 3018-3021.

[42] Segawa, Y.; Miyamoto, S.; Omachi, H.; Matsuura, S.; Šenel, P.; Sasamori, T.; Tokitoh, N.; Itami, K. Concise Synthesis and Crystal Structure of [12]Cycloparaphenylene. Angewandte Chemie International Edition 2011, 50, 3244-3248.

[43] Schweizer, W. B.; Dunitz, J. D. Quantum mechanical calculations for benzene dimer energies: Present problems and future challenges. Journal of Chemical Theory and Computation 2006, 2, 288-291.

[44] Ringer, A. L.; Sherrill, C. D. First principles computation of lattice energies of organic solids: The benzene crystal. Chemistry-A European Journal 2008, 14, 2542-2547.

[45] Beran, G. J.; Nanda, K. Predicting organic crystal lattice energies with chemical accuracy. The Journal of Physical Chemistry Letters 2010, 1, 3480-3487. 
[46] Sancho-García, J. C.; Aragó, J.; Ortí, E.; Olivier, Y. Obtaining the lattice energy of the anthracene crystal by modern yet affordable firstprinciples methods. The Journal of Chemical Physics 2013, 138, 204304.

[47] Sancho-García, J. C.; Pérez-Jiménez, A.; Olivier, Y. Determining the cohesive energy of coronene by dispersion-corrected DFT methods: Periodic boundary conditions vs. molecular pairs. The Journal of Chemical Physics 2015, 142, 054702.

[48] Iwamoto, T.; Watanabe, Y.; Sadahiro, T.; Haino, T.; Yamago, S. SizeSelective Encapsulation of $\mathrm{C}_{60}$ by [10]Cycloparaphenylene: Formation of the Shortest Fullerene-Peapod. Angewandte Chemie International Edition 2011, 50, 8342-8344.

[49] Iwamoto, T.; Watanabe, Y.; Takaya, H.; Haino, T.; Yasuda, N.; Yamago, S. Size-and Orientation-Selective Encapsulation of $\mathrm{C}_{70}$ by $\mathrm{Cy}-$ cloparaphenylenes. Chemistry-A European Journal 2013, 19, 1406114068 .

[50] Iwamoto, T.; Slanina, Z.; Mizorogi, N.; Guo, J.; Akasaka, T.; Nagase, S.; Takaya, H.; Yasuda, N.; Kato, T.; Yamago, S. Partial Charge Transfer in the Shortest Possible Metallofullerene Peapod, $\mathrm{LaC}_{82}$-[11]Cycloparaphenylene. Chemistry-A European Journal 2014, 20, 14403-14409.

[51] Sakamoto, H.; Fujimori, T.; Li, X.; Kaneko, K.; Kan, K.; Ozaki, N.; Hijikata, Y.; Irle, S.; Itami, K. Cycloparaphenylene as a molecular porous carbon solid with uniform pores exhibiting adsorption-induced softness. Chemical Science $\mathbf{2 0 1 6 ,}$ 
[52] Sancho-Garcia, J. C.; Moral, M.; Pérez-Jiménez, A. Effect of Cyclic Topology on Charge-Transfer Properties of Organic Molecular Semiconductors: The Case of Cycloparaphenylene Molecules. The Journal of Physical Chemistry C 2016, 120, 9104-9111.

[53] Roux, M. V.; Temprado, M.; Chickos, J. S.; Nagano, Y. Critically evaluated thermochemical properties of polycyclic aromatic hydrocarbons. Journal of Physical and Chemical Reference Data 2008, 37, 1855-1996.

[54] Ouvrard, C.; Mitchell, J. B. Can we predict lattice energy from molecular structure? Acta Crystallographica Section B: Structural Science 2003, 59, 676-685.

[55] Bagheri, M.; Bagheri, M.; Gandomi, A. H.; Golbraikh, A. Simple yet accurate prediction method for sublimation enthalpies of organic contaminants using their molecular structure. Thermochimica Acta 2012, $543,96-106$. 
- Table 1. Unit cell parameters, and other relevant magnitudes, for the set of $[n]$ CPP compounds.

- Table 2. Interaction energies for the set of dimers of $[n] \mathrm{CPP}$ compounds, as calculated at the B3LYP-D3(BJ)/cc-pVTZ level. The table displays the values from the highest (left) to the lowest (right) stability found for every of the weakly bound dimers. 
Table 1:

\begin{tabular}{|c|c|c|c|c|c|c|c|c|}
\hline$[n] \mathrm{CPP}$ & $\begin{array}{c}a \\
(\AA) \\
\end{array}$ & $\begin{array}{c}b \\
(\AA) \\
\end{array}$ & $\begin{array}{c}c \\
(\AA)\end{array}$ & $\begin{array}{c}\alpha \\
\left(^{\circ}\right)\end{array}$ & $\begin{array}{c}\beta \\
\left(^{\circ}\right)\end{array}$ & $\begin{array}{c}\gamma \\
\left({ }^{\circ}\right)\end{array}$ & $\begin{array}{c}\text { Diameter } \\
(\AA)\end{array}$ & $\begin{array}{c}\text { Density } \\
\left(\mathrm{Mg} \cdot \mathrm{m}^{-3}\right)\end{array}$ \\
\hline$n=5$ & 9.8337 & 11.6263 & 35.5613 & 90 & 90 & 90 & 6.81 & 1.243 \\
\hline$n=6$ & 19.3957 & 19.3957 & 6.1998 & 90 & 90 & 120 & 8.37 & 1.126 \\
\hline$n=7$ & 18.3407 & 22.3358 & 8.2183 & 90 & 90 & 90 & 9.79 & $1.217^{a}$ \\
\hline$n=8$ & 12.9325 & 8.01030 & 19.3676 & 90 & 105.363 & 90 & 11.26 & 1.045 \\
\hline$n=10$ & 15.9075 & 8.1405 & 20.7418 & 90 & 108.655 & 90 & 13.74 & 0.993 \\
\hline$n=12$ & 37.1654 & 16.3756 & 23.6701 & 90 & 106.157 & 90 & 16.56 & $1.038^{b}$ \\
\hline
\end{tabular}
(cyclohexane) molecule.

${ }^{b}$ For a compound with stoichiometry $\mathrm{C}_{84} \mathrm{H}_{72}$, and thus with cocrystallized solvent (cyclohexane) molecules. 
Table 2:

\begin{tabular}{llllll}
\hline$[n] \mathrm{CPP}$ & \multicolumn{5}{c}{$\Delta E_{\mathrm{int}}(\mathrm{kcal} / \mathrm{mol})$} \\
\hline$n=5$ & -12.01 & -6.32 & -5.95 & -3.50 & \\
$n=6$ & -14.21 & -7.90 & -7.86 & -4.60 & \\
$n=7$ & -11.31 & -9.35 & -8.67 & -5.90 & -5.87 \\
$n=8$ & -14.04 & -10.72 & -10.40 & -6.42 & -6.41 \\
$n=10$ & -17.32 & -14.38 & -9.39 & -6.98 & \\
$n=12$ & -18.47 & -16.13 & -7.98 & -7.49 & -7.49 \\
\hline
\end{tabular}


- Figure 1. Chemical structure of the investigated $[n] \mathrm{CPP}$ compounds.

- Figure 2. Interaction energies of all unique dimers found for the $[n] \mathrm{CPP}$, as calculated at the B3LYP-D3(BJ)/cc-pVTZ level: (a) [5]CPP, (b) $[6] \mathrm{CPP},(\mathrm{c})[7] \mathrm{CPP},(\mathrm{d})[8] \mathrm{CPP},(\mathrm{e})[10] \mathrm{CPP}$, and (f) [12]CPP. The dashed line is a guide to the eye.

- Figure 3. Supramolecular arrangement of [6]CPP in the crystalline state. The $\mathrm{H}$ atoms and corresponding $\mathrm{C}-\mathrm{H}$ bonds have been omitted for clarity. The reference molecule, from which the number of uniquely symmetry-interacting dimers is determined, is blue-coloured.

- Figure 4. Supramolecular arrangement of [12]CPP in the crystalline state. The $\mathrm{H}$ atoms and corresponding $\mathrm{C}-\mathrm{H}$ bonds have been omitted for clarity. The reference molecule, from which the number of uniquely symmetry-interacting dimers is determined, is blue-coloured. 


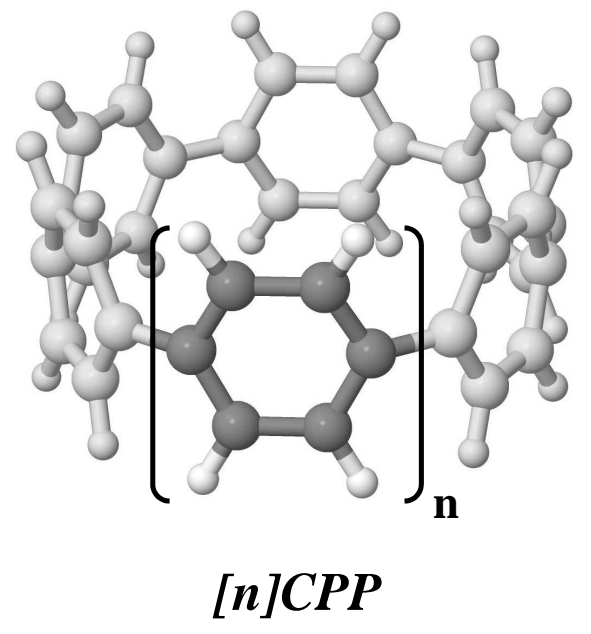

Figure 1. 


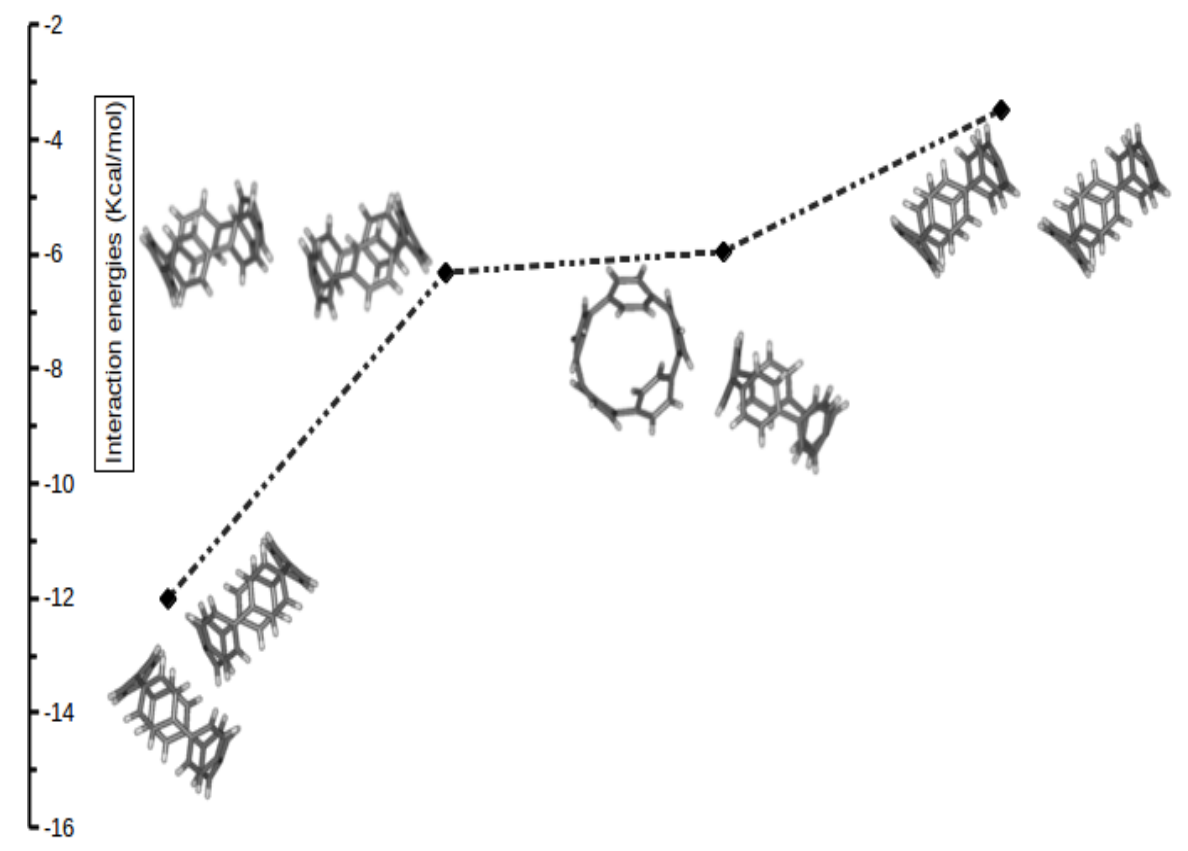

(a) $[5] \mathrm{CPP}$

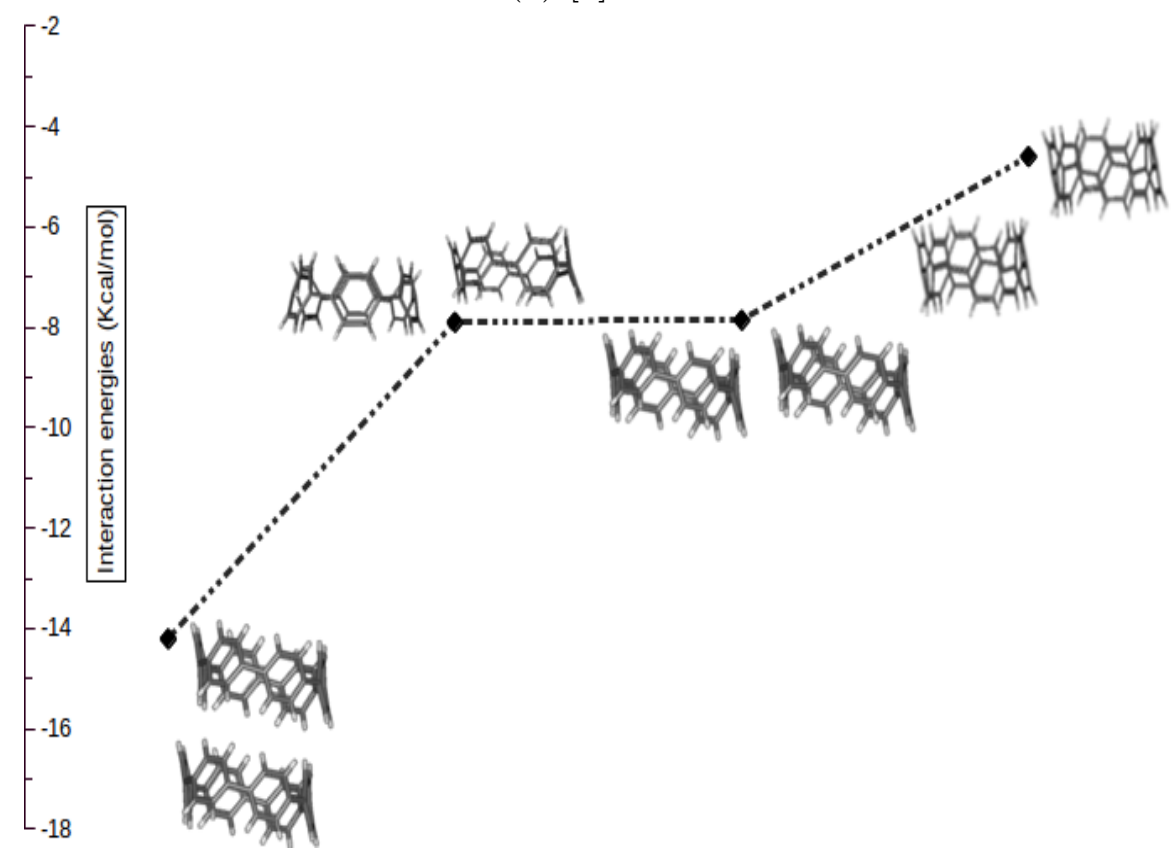

(b) $[6] \mathrm{CPP}$

Figure 2. 


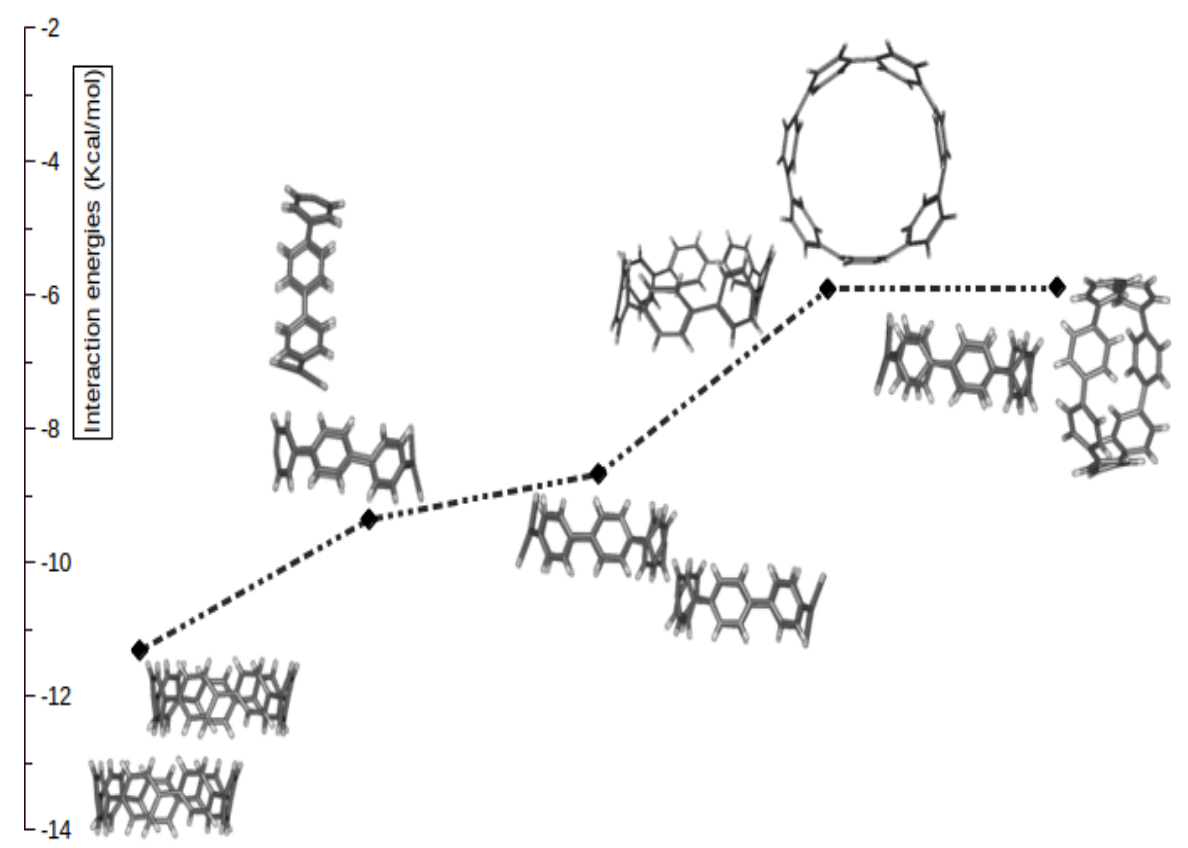

(c) $[7] \mathrm{CPP}$

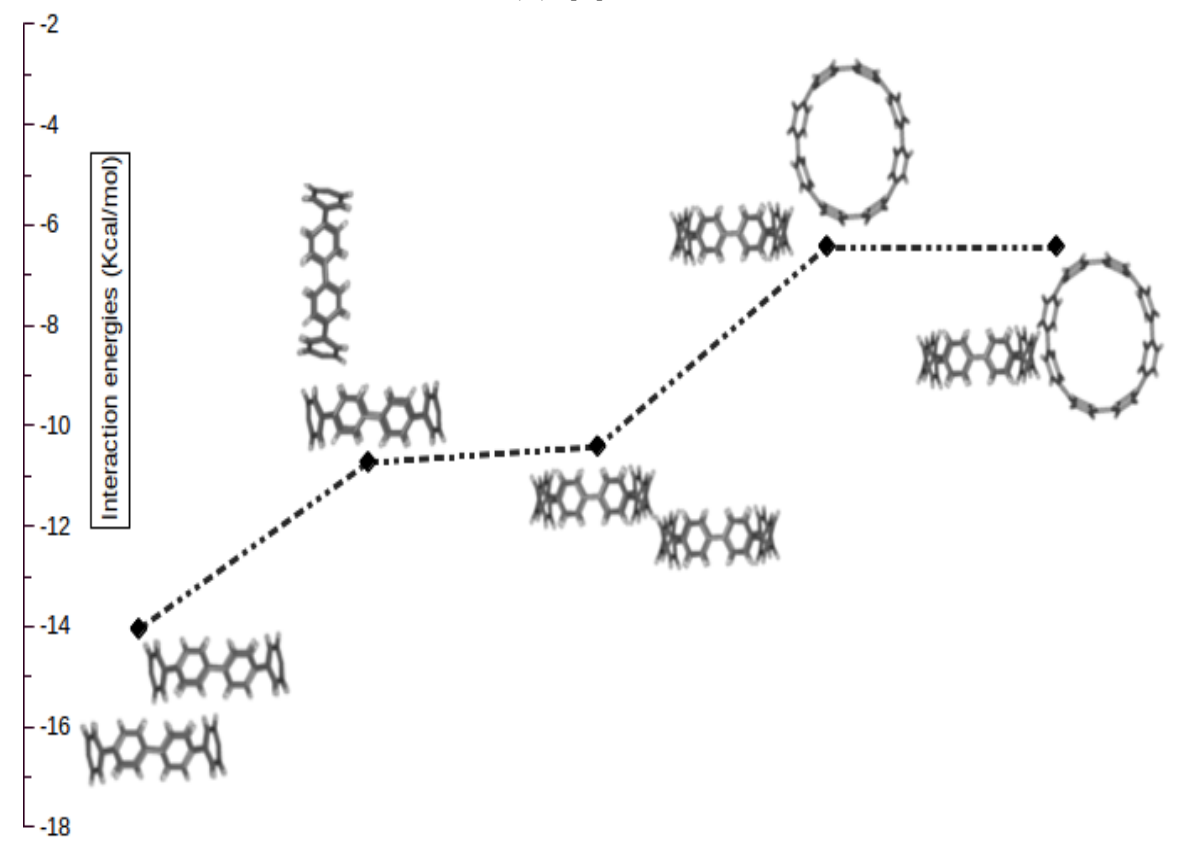

(d) $[8] \mathrm{CPP}$

Figure 2 (cont.) 


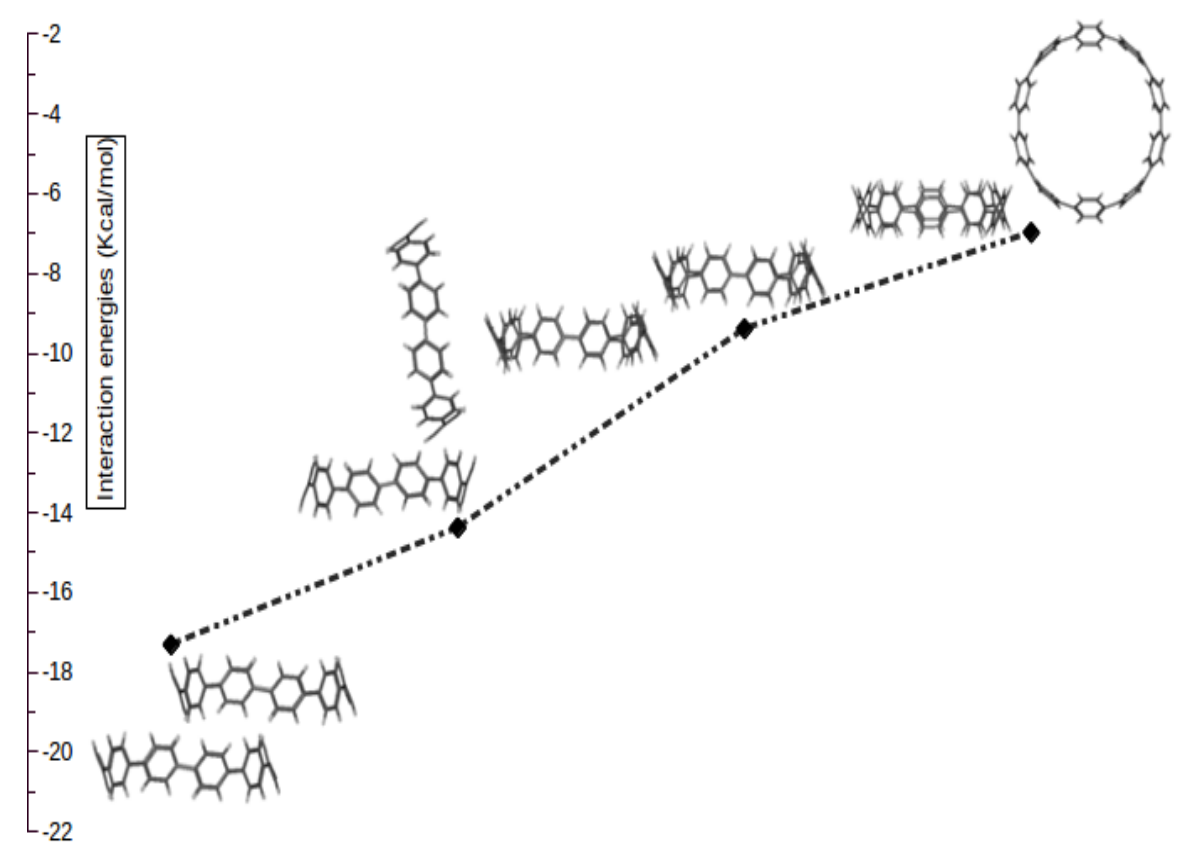

(e) $[10] \mathrm{CPP}$

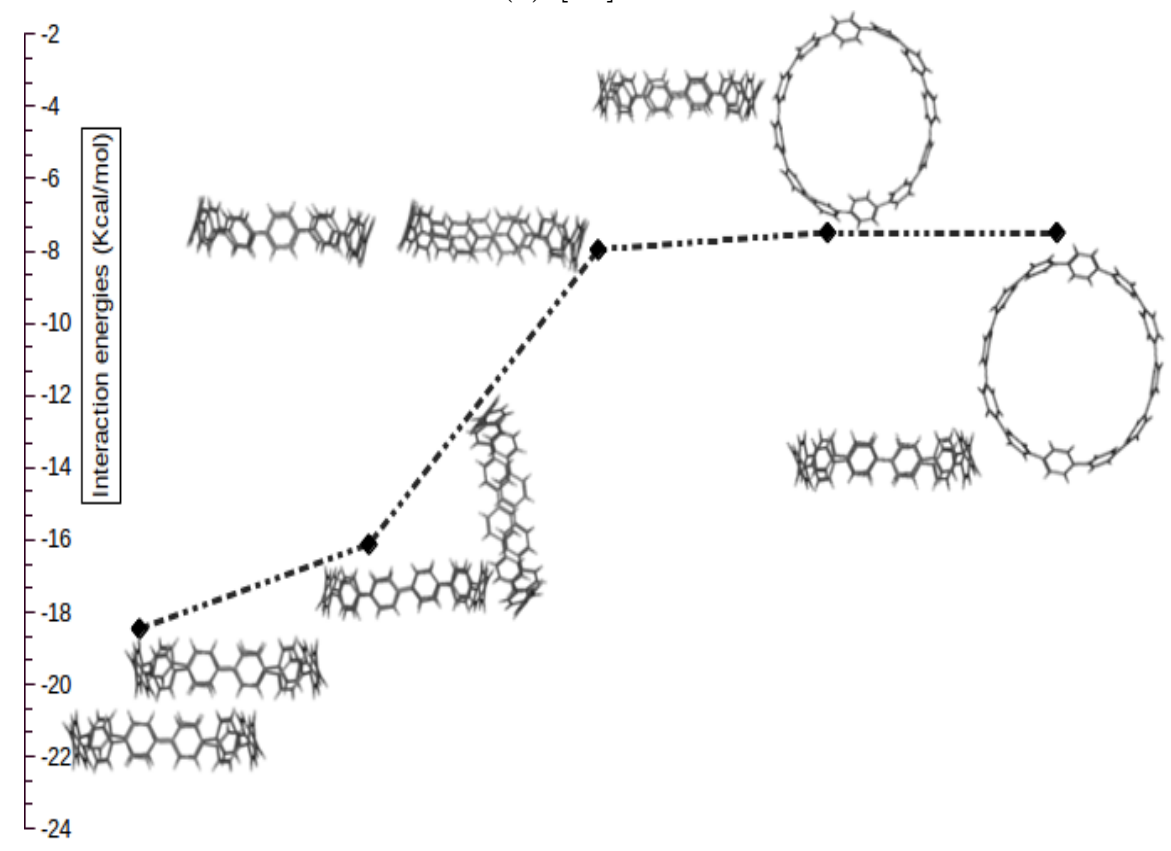

(f) $[12] \mathrm{CPP}$

Figure 2 (cont.) 


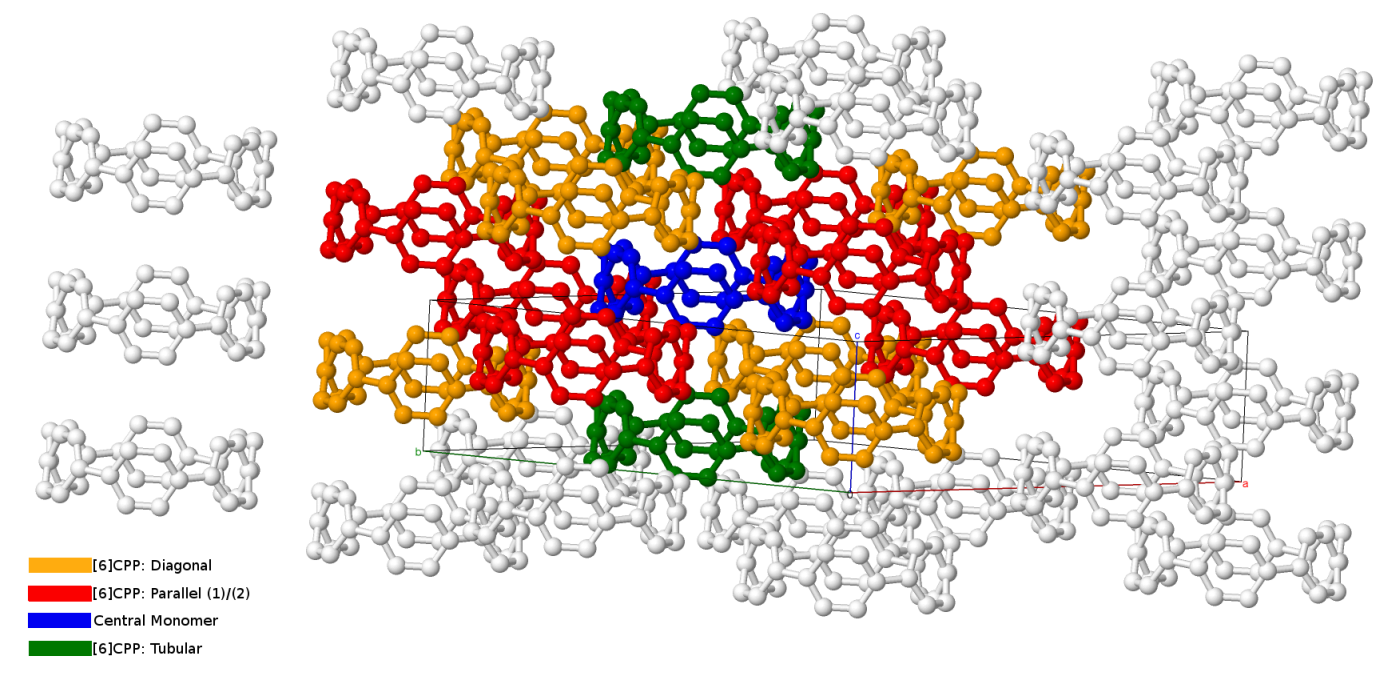

Figure 3. 


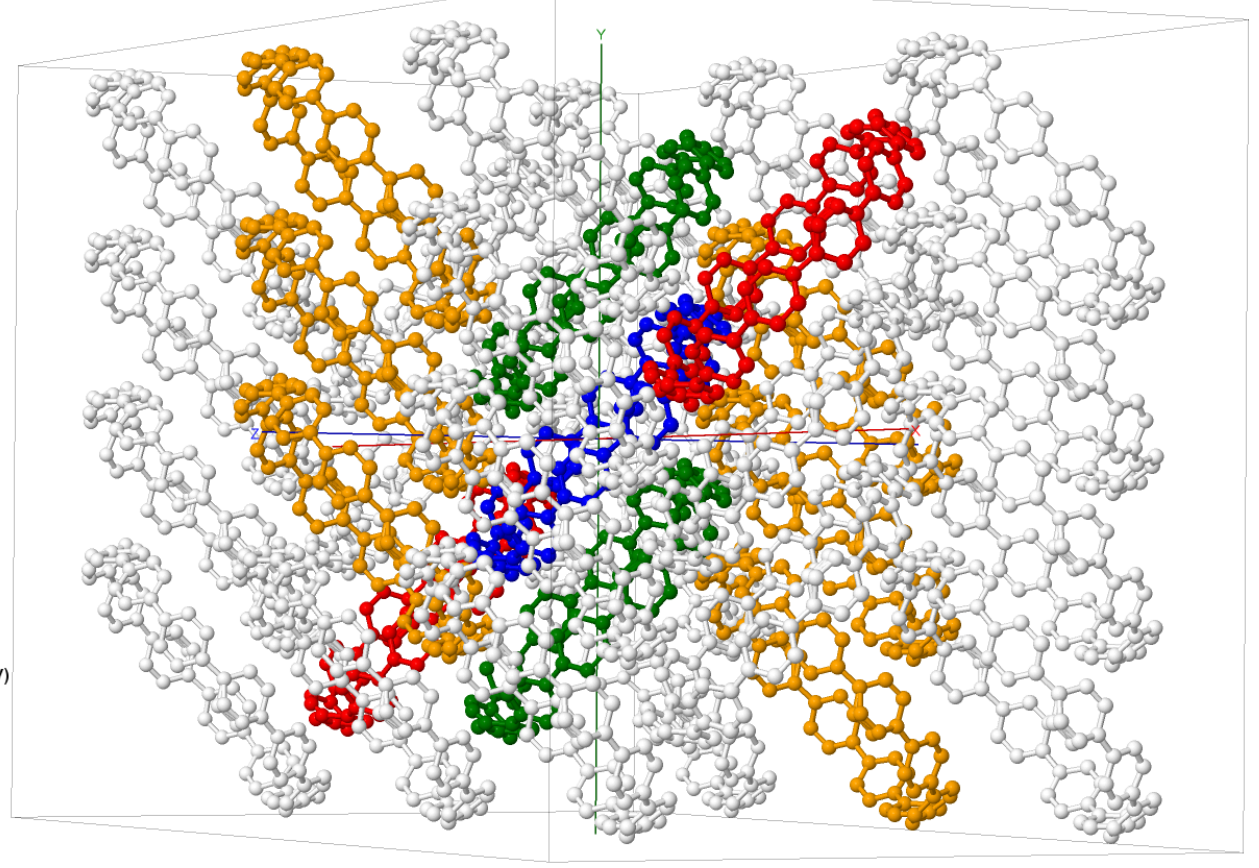

Figure 4. 